\title{
Lipoblastoma retroperitoneal gigante. Caso clínico
} Giant retroperitoneal lipoblastoma. Case report

\author{
Dr. Nicolás I. Rosièrea , Dra. Alejandra G. Falcioni ${ }^{a}$ Dr. Daniel Navacchia ${ }^{a}$ y Dr. Daniel Giambini ${ }^{a}$
}

\section{RESUMEN}

El lipoblastoma forma parte de los tumores derivados del tejido adiposo. Es una neoplasia benigna que se puede presentar de forma localizada o difusa, que afecta a niños en la primera infancia y que, generalmente, se localiza en el tronco y las extremidades.

Estos tumores son poco frecuentes en la edad pediátrica, pero cuando uno de ellos se presenta, constituye un desafío que se debe resolver rápidamente por el riesgo de malignidad que implican otras lesiones neoplásicas, de similares características. El diagnóstico preoperatorio es dificultoso, ya que los métodos por imágenes no aportan información específica que permita diferenciarlos de otros tumores, como los liposarcomas. El diagnóstico definitivo es anatomopalógicoy, en casos dudosos, es necesaria la confirmación citogenética.

Se presenta el caso de un niño de 14 meses de edad con diagnóstico de lipoblastoma de localización retroperitoneal de gran tamaño.

Palabras clave: neoplasias retroperitoneales, lipoblastoma, pediatría.

\begin{abstract}
Lipoblastoma is part of tumors derived from adipose tissue. It is a benign neoplasm that can be localized or diffuse, affecting children in early childhood and usually located in the trunk and extremities.

These tumors are uncommon in the pediatric age, but when one of them is present, it is a challenge that we must resolve quickly due to the risk of malignancy that involves other similar neoplastic lesions.

The preoperative diagnosis is difficult, because the imaging methods do not provide specific information that allows us to differentiate them from other tumors, such as liposarcomas. The definitive diagnosis is anatomopathological and, in doubtful cases, cytogenetic confirmation is necessary.

We present the case of a 14-month-old boy with diagnosis of large sized lipoblastoma of retroperitoneal localization.

Key words: retroperitoneal neoplasms, pediatrics, lipoblastoma.
\end{abstract}

http:/ / dx.doi.org/10.5546/ aap.2018.e139

Cómo citar: Rosière NI, Falcioni AG, Navacchia D, et al. Lipoblastoma retroperitoneal gigante. Caso clínico. Arch Argent Pediatr 2018; 116(1):e139-e142.

a. Hospital Pedro de Elizalde,

Ciudad Autónoma de Buenos Aires.

Correspondencia:

Dr. Nicolás I. Rosière: nicolasirosiere@gmail.com

Financiamiento: Ninguno.

Conflicto de intereses: Ninguno que declarar.

Recibido: 4-6-2017

Aceptado: 15-8-2017

\section{INTRODUCCIÓN}

Los tumores adiposos se clasifican como benignos: lipoma, lipoma heterotópico, lipomatosis, hibernoma y variantes de lipoma, como lipoblastoma y lipoblastomatosis (LBS); o malignos: liposarcoma, que es extremadamente raro en la infancia. ${ }^{1}$

El lipoblastoma forma parte de los tumores de tejido blando derivado de la proliferación posnatal de la grasa embrionaria y constituye una neoplasia benigna e infrecuente que se desarrolla en lactantes y niños. Este término fue utilizado por primera vez en 1926 por Jaffe, quien lo describió como un tumor de células grasas inmaduras. ${ }^{2}$ En 1973, Chung y Enzinger definieron el lipoblastoma como una neoplasia localizada y la lipoblastomatosis como la expresión difusa de la misma patología. ${ }^{3}$

La etiopatogenia de los tumores benignos adiposos aún no está clara. E1 70\% de los lipoblastomas presenta un reordenamiento del oncogén PLAG1 en el cromosoma 8q11-13 y hasta un $18 \%$ tiene una polisomía para este cromosoma. El gen PLAG1 está implicado en la mitogénesis celular, la proliferación, la apoptosis y la regulación positiva del factor de crecimiento insulínico tipo $2 .{ }^{1}$

Si bien se trata de un tumor benigno, aun con los avances actuales en los métodos de diagnóstico por imágenes, no es posible diferenciarlo de lesiones malignas, como el liposarcoma. ${ }^{4}$ Por esta razón, constituye un desafío para el equipo médico tratante que debe ser resuelto de manera rápida y eficaz.

A continuación, se comparte un caso de lipoblastoma en un lactante, no solo por la baja incidencia de esta patología, sino también por su gran tamaño e infrecuente ubicación.

\section{REPORTE DE CASO}

Niño de 14 meses de edad, de $11 \mathrm{~kg}$ de peso, previamente sano y sin antecedentes personales de importancia, que consulta por masa abdominal palpable de un mes de evolución, sin síntomas asociados. Al momento del examen físico, presenta una lesión indurada, de gran tamaño y fija en la región umbilical, flanco y fosa 
ilíaca derecha (Figura 1).

La ecografía abdominal informa masa heterogénea de contornos lobulados, retroperitoneal derecha que sobrepasa la línea media, de $190 \times 96 \times 157 \mathrm{~mm}$.

El análisis de laboratorio informa hemoglobina: 8,9 g/ dl (valor normal -VN-: 10,5); hematocrito: $30 \%$ (VN: 33); potasio sérico: $5,8 \mathrm{mEq} / \mathrm{L}$ (VN: 3,1-5,1); lactato deshidrogenasa (LDH): $644 \mathrm{UI} / \mathrm{L}$ (VN: 230-480); hormona gonadotropina coriónica humana (BHCG): 2,8 $\mathrm{mUI} / \mathrm{ml}(\mathrm{VN}: 0-1,5)$. El resto de los estudios están dentro de los límites normales.

En la tomografía computada (TC) con contraste oral y endovenoso, se observa una masa

FIgura 1. Paciente en la mesa quirúrgica, palpación y delimitación de la lesión preoperatoria

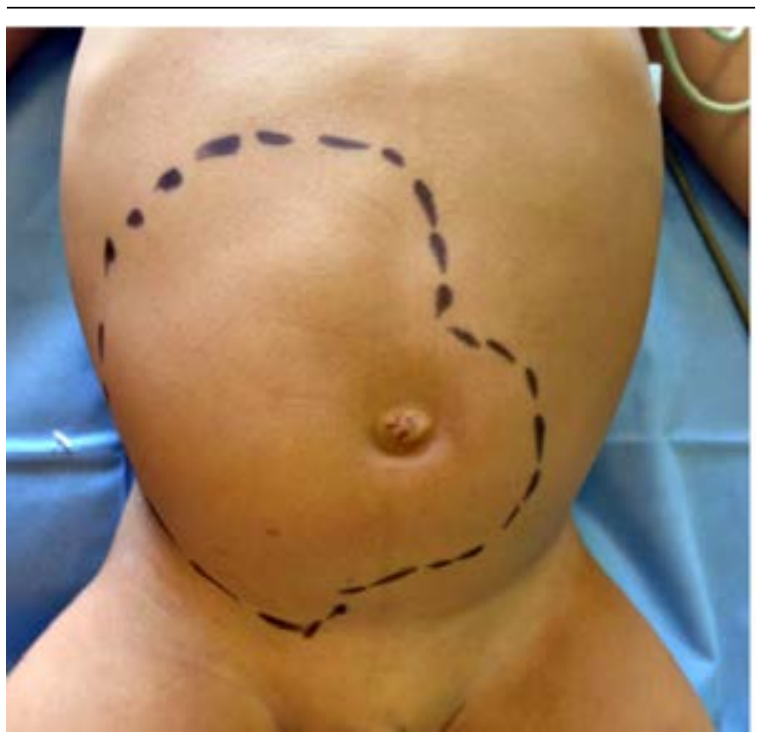

retroperitoneal multilobulada, heterogénea, con predominio de áreas de densidad grasa (-90 UH) y discreto componente sólido, el cual presenta realce ante el contraste endovenoso. Diámetros: 130 x 163 × 93 mm (Figura 2). Diagnóstico presuntivo: lipoblastoma versus liposarcoma.

Se decide la conducta quirúrgica (Figura 3). Se realiza una incisión trasversa supraumbilical derecha y exéresis de masa retroperitoneal en tres fragmentos, que, en su totalidad, mide $160 \times 120 \mathrm{x}$ $80 \mathrm{~mm}$ y pesa 469 gramos (Figura 3). La anatomía patológica informa neoplasia benigna compuesta por lipoblastos con septos fibroconectivos y una fina red vascular, sin evidencia de atipia celular (Figura 4).

El paciente presenta íleo posoperatorio de 6 días de evolución, que se resuelve con ayuno, nutrición parenteral y sonda nasogástrica descompresiva. $\mathrm{Al} 10^{\circ}$ día, se otorga el egreso hospitalario. Se realizan controles ecográficos mensuales los primeros 6 meses posoperatorios, sin evidenciar imágenes patológicas. Actualmente, se encuentra en seguimiento trimestral.

\section{DISCUSIÓN}

El lipoblastoma se manifiesta, generalmente, en la infancia, y la edad promedio de diagnóstico es a los tres años de edad. ${ }^{1,4,5}$ Se han descrito casos en adultos; sin embargo, son infrecuentes. ${ }^{1}$

Se presenta, con más frecuencia, en varones, como una masa palpable, móvil y no dolorosa, habitualmente, localizada en el tronco y las extremidades (axilas, hombros, codos, manos) $\mathrm{y}$, con menos frecuencia, en la ingle y el cuello. ${ }^{1,4}$ Las formas retroperitoneales son infrecuentes y asintomáticas, por lo cual, generalmente, adquieren gran tamaño. ${ }^{1,45}$ La sintomatología

FIGURA 2.Tomografía computada con contraste oral y endovenoso: masa retroperitoneal multilobulada, heterogénea, con predominio de áreas de densidad grasa (-90 UH) y discreto componente sólido, la cual presenta realce ante el contraste endovenoso. Diámetros: $130 \times 163 \times 93 \mathrm{~mm}$




que desarrollen dependerá del desplazamiento o la compresión de las estructuras vecinas, con el crecimiento progresivo del tumor.

Ante una masa de estas características, se plantean diversos diagnósticos diferenciales dependiendo de su localización. Aquellos que se presentan en las extremidades deben ser diferenciados del liposarcoma, rabdomiosarcoma, sarcoma de células claras, tumores desmoides, sarcoma epitelioide, sarcoma sinovial, lipomas intramusculares, angiolipomas y hemangiomas. Si la ubicación es una masa abdominal, debe considerarse la posibilidad de otros tumores, como teratoma, nefroblastoma, neuroblastoma, quistes de mesenterio y epiplón. En la región inguinal, se debe considerar el quiste de inclusión epidérmica y el testículo ectópico. A nivel torácico, se debe descartar mama supernumeraria, linfoma y mixoma auricular.
El diagnóstico preoperatorio es poco frecuente. ${ }^{5,6}$ La principal dificultad diagnóstica se presenta entre lipoblastoma y lipoma o liposarcoma, ya que, radiológicamente, son neoplasias muy similares, pero de pronóstico muy diferente.

Los estudios de imágenes, como TC y resonancia magnética nuclear $(\mathrm{RMN})$, son útiles para realizar una precisa ubicación topográfica de la lesión y planificación quirúrgica. No permiten hacer un adecuado diagnóstico diferencial entre las diferentes neoplasias de tejido graso. ${ }^{6-9} \mathrm{La}$ RMN sigue siendo el método más sensible para el diagnóstico; el aumento de la vascularización en los lipoblastomas, en comparación con los lipomas, muestra una menor intensidad en las imágenes ponderadas $\mathrm{T} 1 .^{9}$

La citología por punción y aspiración con agua fina es un método diagnóstico que está descrito

FIGURA 3. Laparotomía: la lesión retroperitoneal desplaza el colon ascendente y transverso hacia el lado izquierdo (flecha). Pieza quirúrgica sólida, capsulada, multilobulada, coloración amarillenta. Mide $16 \times 12$ x 38 cm y pesa 469 gramos
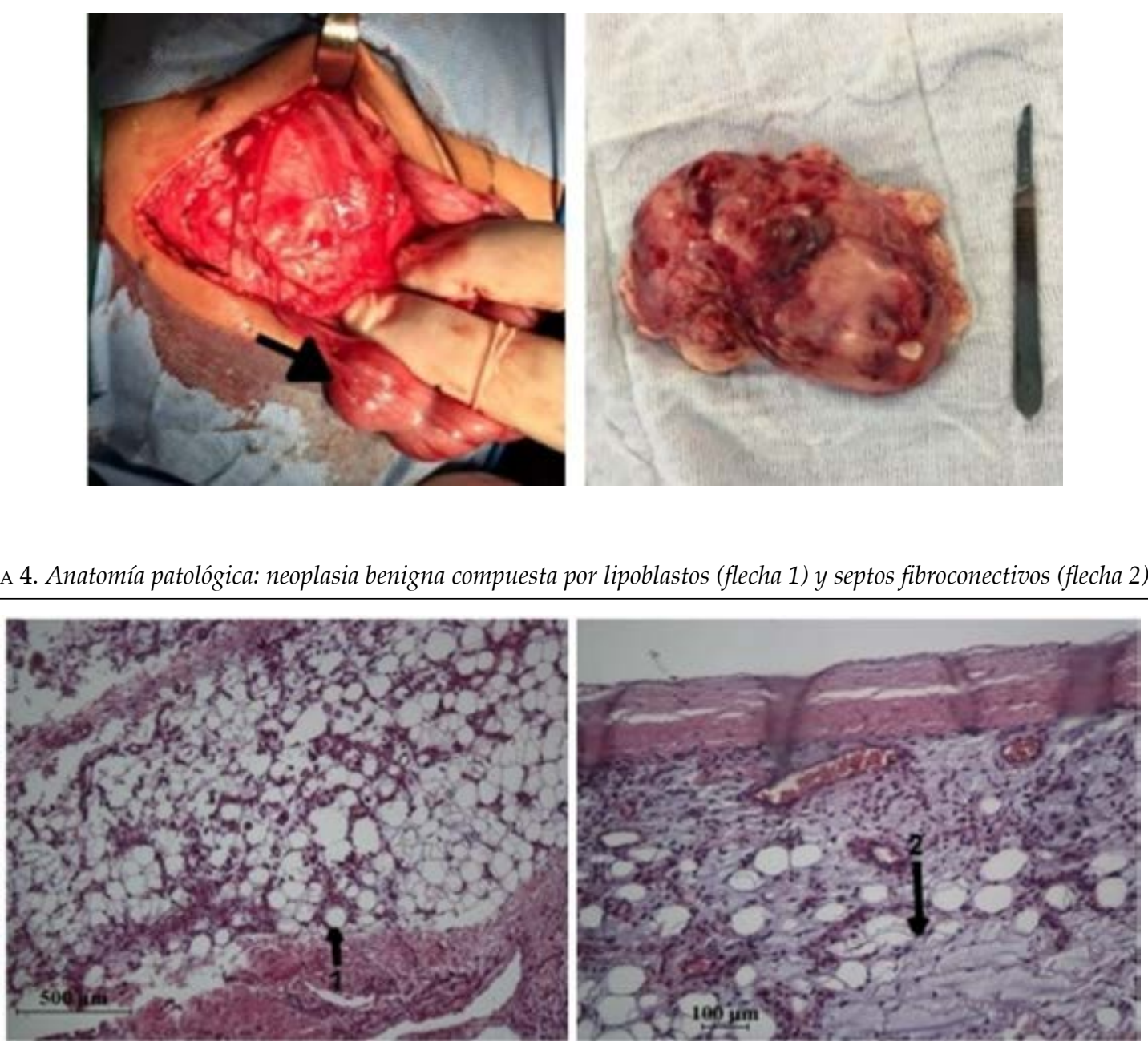
en pocas series. En los hallazgos, suele ser difícil diferenciar un lipoblastoma de un lipoma. ${ }^{4,9}$

La anatomía patológica confirma el origen de la lesión. El examen de la pieza muestra que se trata de tumores encapsulados, lobulados, adipocitos de localización central, lipoblastos dispersos periféricamente, estroma mixoide con septos fibrosos y una prominente red capilar. Los lipoblastos se caracterizan por células con abundantes vacuolas grasas en su citoplasma, carentes de atipia celular.

En ocasiones, el diagnóstico diferencial con otros tumores suele ser dificultoso, como, por ejemplo, con liposarcomas bien diferenciados o mixoides. ${ }^{1,10}$ Estos últimos son extremadamente infrecuentes, compuestos de lipoblastos de aspecto fusiforme, una matriz mixoide, septos vascularizados y aspecto lobulado, pero menos marcado que en el lipoblastoma; además, se evidencia atipia celular. En estos casos, el análisis citogenético constituye una herramienta útil para llegar al diagnóstico certero. Reordenamientos cromosómicos 8q11-13 son diagnósticos de lipoblastoma, mientras que una translocación cromosomal 12-16 se observa en el liposarcoma mixoide. ${ }^{14,11,12}$ La evaluación citogenética también puede ser útil para diferenciar lipoma de lipoblastoma. El lipoma, típicamente, muestra diferentes reordenamientos del cromosoma 12 (q13-q15), que resultan en la desregulación del gen $H M G A 2{ }^{1}$

El tratamiento de elección es la exéresis completa. El procedimiento quirúrgico no debe ser mutilante. En aquellos casos en los cuales la extirpación tumoral no se realiza de forma completa, se propone realizar un enfoque por etapas y reevaluar la posibilidad de realizar un nuevo abordaje quirúrgico desde otra incisión. Se ha descrito, en la literatura, la conducta expectante y la regresión espontánea ante la presencia de lesiones en las cuales el tratamiento quirúrgico sería mutilante. ${ }^{13}$

El índice de recurrencia varía según las diferentes series de $0 \%$ a $25 \%$. El tiempo de seguimiento es controversial; se sugiere de uno a tres años. ${ }^{5,7,8}$ La recidiva aumenta en el caso de lipoblastomatosis. Estos se presentan hasta 84 meses después de la resección, generalmente, dentro de los 2 primeros años de seguimiento, por lo que se recomienda una vigilancia posoperatoria constante de, al menos, 5 años: cada 2 meses durante el primer año, cada 6 durante el segundo y tercer año y luego de forma anual. En aquellos que presentan múltiples recurrencias o resecciones incompletas, se propone un seguimiento más prolongado con estudios por imágenes periódicas, como RMN. ${ }^{14}$ No se han descrito las metástasis a la fecha. ${ }^{1,15}$

\section{REFERENCIAS}

1. Cascini V, Lisi G, Lauriti G, et al. Giant abdomino-pelvic adipose tumors of childhood. Pediatr Surg Int 2012; 28(1):89-93.

2. Jaffe RH. Recurrent lipomatous tumors of the groin: liposarcoma and lipoma pseudomyxomatodes. Arch Pathol 1926;1:381-7.

3. Vellios F, Baez J, Shumacker HB. Lipoblastomatosis: a tumor of fetal fat different from hibernoma: a report of a case, with observations on the embryogenesis of human adipose tissue. Am J Pathol 1958;34(6):1149-59.

4. Ghosh P, Das RN, Ghosh R, et al. Lipoblastoma and lipoblastomatosis: A clinicopathological study of six cases. J Cancer Res Ther 2015;11(4):1040.

5. Speer AL, Schofield DE, Wangc KS, et al. Contemporary management of lipoblastoma. J Pediatr Surg 2008;43(7): 1295-300.

6. McVay MR, Keller JE, Wagner CW, et al. Surgical management of lipoblastoma. J Pediatr Surg 2006;41(6): 1067-71.

7. Dilley AV, Patel DL, Hicks MJ, et al. Lipoblastoma: pathophysiology and surgical management. J Pediatr Surg 2001;36(1):229-31.

8. Stringel G, Shandling B, Mancer K, et al. Lipoblastoma in infants and children. J Pediatr Surg 1982;17(3):277-80.

9. Burchhardt D, Fallon SC, Lopez M, et al. Retroperitoneal lipoblastoma: A discussion of current management. JPediat Surg 2012;47(10):e51-4.

10. Chung EB, Enzinger FM. Benign lipoblastomatosis. An analysis of 35 cases. Cancer 1973;32(2):482-92.

11. Fletcher CD, Akerman M, Dal Cin P, et al. Correlation between clinicopathological features and karyotype in lipomatous tumors. A report of 178 cases from the Chromosomes and Morphology (CHAMP) Collaborative Study Group. Am J Pathol 1996;148(2):623-30.

12. Miller GG, Yanchar NL, Magee JF, et al. Lipoblastoma and liposarcoma in children: an analysis of 9 cases and a review of the literature. Can J Surg 1998;41(6):455-8.

13. Mognato G, Cecchetto G, Carli M, et al. Is surgical treatment of lipoblastoma always necessary? J Pediatr Surg 2000;35(10):1511-3.

14. ValderramaJ,SchnettlerD,ZamoranoM, etal.Lipoblastoma retroperitoneal en un lactante. Caso clínico. Rev Chil Pediatr 2015;87(3):199-203.

15. Mentzel T, Calonje E, Fletcher CDM. Lipoblastoma and lipoblastomatosis: a clinicopathological study of 14 cases. Histopathology 1993;23(6):527-33. 